\title{
Tuberculosis among Chronic Hemodialysis Patients: A Senegalese Single Center Experience
}

\author{
Mouhamadou Moustapha Cisse1, Rachid El Kabouss', Yaya Kane², Sidy Mouhamed Seck3, \\ Ahmed Tall Lemrabott ${ }^{1}$, Maria Faye ${ }^{1}$, El Hadji Fary Ka ${ }^{1}$, Ansoumana Diatta ${ }^{3}$, \\ Abdou Niang1, Boucar Diouf ${ }^{1}$ \\ ${ }^{1}$ Nephrology Department of Teaching, Hospital Aristide le Dantec, Dakar, Senegal \\ ${ }^{2}$ Department of Nephrology, Assane Seck University, Ziguinchor, Senegal \\ ${ }^{3}$ Department of Nephrology, Gaston Berger University, Saint Louis, Senegal \\ Email: "mhmcisse@yahoo.fr
}

Received 1 November 2015; accepted 18 December 2015; published 21 December 2015

Copyright (C) 2015 by authors and Scientific Research Publishing Inc.

This work is licensed under the Creative Commons Attribution International License (CC BY). http://creativecommons.org/licenses/by/4.0/

(c) (i) Open Access

\section{Abstract}

Summary: Tuberculosis is a common infectious disease in chronic hemodialysis due to alteration of the immune system associated with chronic kidney disease. The objectives of this study are to determine the prevalence of tuberculosis in chronic hemodialysis patients and to identify its diagnostic and therapeutic difficulties. Methods and patients: This was a descriptive retrospective study over a period of 20 years (1994-2014). It includes the records of periodic hemodialysis patients in the Nephrology Department of the Aristide Le Dantec University Teaching Hospital in Dakar which clinical symptoms and laboratory favor tuberculosis. Results: Of 258 chronic hemodialysis patients treated in Hospital Aristide Le Dantec hemodialysis center, 29 cases (11.4\%) of tuberculosis disease are diagnosed. The mean age is $43.21 \pm 12.48$ years, and the sex-ratio is 0.8 . The median time to onset of tuberculosis after initiation of hemodialysis is $22.86 \pm 28.86$ months. The diagnosis of tuberculosis is sure only in $17 \%$ of cases. Extra-pulmonary sites are found in $79 \%$ of cases. The average duration of treatment is $9.39 \pm 1.64$ months $(6-13$ months). Various treatment protocols are adopted. Mortality is $21 \%, 50 \%$ due to disseminated tuberculosis. Conclusion: The diagnosis of tuberculosis in the chronic hemodialysis patients is often difficult due to the atypical symptoms, the frequency of extra-pulmonary location and the lack of evidence of sure diagnosis.

\section{Keywords}

Tuberculosis, Hemodialysis, Dakar

\footnotetext{
${ }^{*}$ Corresponding author.
}

How to cite this paper: Cisse, M.M., Kabouss, R.E., Kane, Y., Seck, S.M., Lemrabott, A.T., Faye, M., Ka, E.H.F., Diatta, A., Niang, A. and Diouf, B. (2015) Tuberculosis among Chronic Hemodialysis Patients: A Senegalese Single Center Experience. Open Journal of Nephrology, 5, 117-122. http://dx.doi.org/10.4236/ojneph.2015.54017 


\section{Introduction}

Tuberculosis (TB) is one of the most frequent infectious complications in chronic hemodialysis patients (CHD) due to dysfunction of cell-mediated immunity (CMI), which occurs in chronic kidney disease (CKD) and increases during dialysis [1] [2]. It has several features. First, the symptoms are often atypical; extra pulmonary locations are more frequent [3] and furthermore toxicity of drugs is more pronounced [3] [4]. The objectives of this study are to determine the patterns of tuberculosis in chronic hemodialysis, tolerance of treatment and outcomes.

\section{Patients and Methods}

This was a descriptive retrospective study over a period of 20 years (1994-2014) on the records of 258 patients treated with regular periodic hemodialysis. The eligible patients must have been on dialysis for at least three months. They were dialyzed 2 to 3 times a week with duration of 4 to 6 hours in Hospital Aristide Le Dantec hemodialysis center. Our local ethics committee based on the hospital approved this study. The diagnosis of TB was retained:

- A number of presumptive arguments. Clinical (unexplained persistent fever for more than two weeks, asthenia, anorexia, dry weight loss associated with respiratory symptoms, digestive, osteo-articular), paraclinical: laboratory findings (inflammatory syndrome, hypercalcemia, tuberculin skin test (TST) positive for interferon gamma detection assays (IGRAs) and cyto-chemical analysis of effusion fluid of serosa \pm assay of adenosine deaminase (ADA)); and morphological (standard X-ray, ultrasound, computed tomography, bronchoscopy). The TST is performed by intradermal injection of $0.1 \mathrm{ml}$ of tuberculin purified protein derivative into the inner surface of the forearm. The injection should be made with a tuberculin syringe, with the needle bevel facing upward. The skin test reaction was read 72 hours after administration. Inflammatory syndrome is defined by the elevation of the white blood cells and CRP.

- Sure arguments (bacteriological and/or histological).

First-line antituberculosis drugs were used at doses adapted to renal function: Rifampicin (R) (10 mg/kg/day), isoniazid (H) (5 mg/kg/day), pyrazinamide (Z) (30 mg/kg/48 h), ethambutol (E) (20 mg/kg/48 h) and streptomycin (S) (500 mg, twice a week).

The new TB cases were treated with a four RHZE or SRHE during the attack phase of two-months, followed by a maintenance phase involving HR for a variable period. The retreatment protocol for relapsing cases comprised an initial phase lasting three months involving five antibacillary agents SRHZE for two months, then RHZE for the 3rd month and a maintenance phase involving RHE for five months. Monitoring of treatment included the verification of compliance, efficiency and seeking treatment side effects. Non-compliance was assessed by both a self-reported survey and an inspection of remaining tablets. For each record, we collected epidemiological (age, gender, history of TB, initial nephropathy, date of first hemodialysis, immunization against TB), clinical (weight, fever, anorexia, clinical exam etc.), paraclinical, therapeutic and outcome data. The collected data were entered and analyzed statistically using the SPSS 20.0.

\section{Results}

Of 258 regular hemodialysis patients treated in Hospital Aristide Le Dantec hemodialysis center, 29 (11.4\%) cases of TB disease were collected. The mean age was $43.21 \pm 12.48$ years $(18-73)$ and the sex ratio $(\mathrm{M} / \mathrm{F})$ was 0.8 . The history of treated TB before initiation of hemodialysis was found in $7 \%$ of cases. Vascular nephropathy (38\%), glomerular (28\%) and polycystic kidney disease (10\%) were the most common causes of CKD. The average time between the initiation of hemodialysis and diagnosis of TB was $22.86 \pm 28.86$ months (0-108 months). Seventeen patients (59\%) developed TB during the first year of dialysis. The main general signs found were unexplained prolonged fever (90\%), anorexia (76\%) and decreasing dry weight (62\%). Respiratory symptoms were the most noted. These were cough (62\%), chest pain (45\%), and dyspnea (45\%). On physical examination, the pleural effusion was found in $40 \%$ of cases, as cites in $14 \%$ of cases, cervical lymphadenopathy in $7 \%$ and pericardial effusion in $3 \%$ of cases.

The biological inflammatory syndrome was noted in all patients. The CRP average was $155.62 \pm 152.37 \mathrm{mg} / \mathrm{l}$. Anemia was observed in $42 \%$ of patients, the mean hemoglobin was $8.56 \pm 1.5 \mathrm{~g} / \mathrm{dl}$. Lymphopenia was observed in $34 \%$ of cases. Mean serum calcium was $93.86 \pm 8.72 \mathrm{mg} / \mathrm{l}$; it was high in $7 \%$ of cases. The mean serum albumin was $33 \pm 5.13 \mathrm{~g} / \mathrm{l}$; it was low in $21 \%$ of cases. The tuberculin skin test was negative in $76 \%$ of cases. 
Tests interferon release assays Y (IGRAs) were performed in six patients, they were positive in $100 \%$ of those cases. Twenty-one of the serous effusions were studied, cytochemical analysis showed a high exudate cells. The mean albumin level in serous effusion was $41.78 \pm 7.65 \mathrm{~g} / \mathrm{l}$. The average rate of lymphocytes was $74.13 \pm$ $11.82 \%$. Adenosine deaminase (ADA) was measured in three patients, it was positive in two cases. The chest radiograph was systematic; it showed a pathological appearance in $75 \%$ of cases. CT scan done in five patients was pathological in all cases. The diagnosis of tuberculosis was sure in $17 \%$ of cases; $7 \%$ to the bacteriology, $7 \%$ at histology and 3\% at autopsy. Extra-pulmonary tuberculosis was noted in $79 \%$ of cases. The tuberculosis locations are summarized in Table 1.

According to the therapeutic patterns, the average duration of treatment was $9.39 \pm 1.64$ months, with a range of 6 to 13 months (6 months for first cases and more for relapses).

Side effects (SE) were reported in $48 \%$ of patients. They were linked to $\mathrm{H}$ in $60 \%$ of cases, $\mathrm{Z}$ in $20 \%$ of cases, $\mathrm{S}$ in $13 \%$ of cases and $\mathrm{E}$ in $7 \%$ of cases. No SE was reported with R. The noted side effects are reported in Table 2.

These SE regressed after therapeutic adjustment. Non-compliance with treatment was reported in $13 \%$ of cases. The treatment was extended with education for good adherence. The outcome was positive in $72 \%$ of cases. Relapse of tuberculosis was reported in $9.5 \%$ of cases. The outcome was positive after new treatment in all cases. Treatment is ongoing in $7 \%$ of cases. The mortality rate was $21 \%$. It was attributed to disseminated TB in $50 \%$ of cases, cardiovascular comorbidity in $33 \%$ of cases and sudden death in $17 \%$ of cases.

\section{Discussion}

The prevalence of TB in the CHD in our series was $11.4 \%$. In the literature it is variously appreciated. It is similar to that reported in a previous study in Senegal [3]. It corroborates those found in Tunisia, Brazil and India, where it was respectively 10\%, 10.3\% and 10.5\% [5]-[7]. A higher prevalence (15.5\%) was reported in Mali [8]. However, low prevalence was reported in Turkey, Ivory Coast and Morocco with respectively 5.2\%, 5.9\% and 6\% [9]-[11]. In developed countries, low prevalence was noted in the US and Japan; it was respectively $1.6 \%$ and $4.93 \%$ [12] [13], however, in Belgium it was high in the range of $15 \%$ and it was attached to an immigration factor [14].

Table 1. TB sites in our study.

\begin{tabular}{lll}
\hline Localisation of TB & Numbers & Percentage \% \\
\hline Pulmonary & $\mathbf{6}$ & $\mathbf{2 1}$ \\
Extra-Pulmonary & $\mathbf{2 3}$ & $\mathbf{7 9}$ \\
Pleural & 10 & 34 \\
Peritonal & 5 & 17 \\
Lymphnodes & 3 & 10 \\
Spondylodiscitis & 1 & 4 \\
Genito-urinary system & 1 & 4 \\
Multifocal & 3 & 10 \\
\hline
\end{tabular}

Table 2. Reported side effects (SE).

\begin{tabular}{llll}
\hline SE & Number of patients & Suspected anti TB & Management \\
\hline LL paresthesia & 5 & H & Vitamin B \\
Acute delirium & 3 & H & Decrease dose of Vitamin B \\
Drug-induced hepatitis & 1 & H & Decrease the dose \\
Hyperuricemia & 3 & Z & Stop Z \\
Hypoacousia & 2 & S & Stop S \\
Decreased visual acuity & 1 & E & Decrease the dose \\
\hline
\end{tabular}


In our series, the prevalence of TB was 56.2 times higher than in the Senegalese general population where it is estimated to be 200/100,000. The high prevalence of TB in the CHD patients compared to the general population was confirmed by other authors [3] [7] [10] [11] [15]. A literature review reported that the incidence is 6.9 to 52.5 times higher in dialysis patients [16]. It is explained by the CMI deficiency related to CKD. TB occurs most often during the first year of the start of hemodialysis. During this phase there is a decrease in CMI, promoting the reactivation of an old or a new TB infection [3] [7] [12]. In our study, the period average was $22.86 \pm$ 28.86 months, 59\% of TB diagnosis was made during the first year after the start of hemodialysis.

TB of dialysis is characterized by its insidious development and nonspecific clinical signs [3] [6] [7] [15]. As in our series, unexplained prolonged fever, anorexia and dry weight loss are the most marked signs. The main respiratory symptoms found were cough in $62 \%$ of cases, chest pain and dyspnea. They are often wrongly attributed to the dialysis constraints [3] [6] [9] [11] [15].

As in our study, the presence of a biological inflammatory syndrome is common [3] [5] [9] [11]. Some authors reported hypercalcemia, it would be an early indicator of TB in hemodialysis patients [17] [18], and it was reported in $7 \%$ of our patients.

In our series TST was negative in $76 \%$ of cases, this result corroborates those found by several authors, its negativity reflects the state of anergy secondary to lower CMI [3] [5] [9] [11] [15]. The authors agree on the low diagnostic value of this review in the context of dialysis [3] [6] [11] [15]. To increase the sensitivity of the test, some authors recommend the TST double stages with a 7 to 15 day intervals for patients not responding to the initial test [12] [14], others have proposed that the TST positivity threshold $5 \mathrm{~mm}$ instead of $10 \mathrm{~mm}$ as in HIV positive patients [19] [20].

The use of IGRAs tests in hemodialysis patients is controversial. Savaj [21] found 23.4\% of positive IGRAs tests against 43.5\% positive TST. Maden [22] did not find a significant difference between the two tests. However, other authors have demonstrated the superiority of IGRAs tests compared to the TST in sensitivity and specificity [13] [23].

In our series, 21 serous effusions were analyzed, they found a rich exudate cells in all cases. This result is similar to those reported by some authors [3] [10]. The dosage of the ADA was positive in two of the three patients examined. According to Gobert [24] there is a strong correlation between the positivity of this assay and the existence of TB; it would be more reliable in the ascites than in the pleural effusions. Nevertheless, a positive determination of ADA is not definitive evidence of TB.

Searching Koch Bacilli in biological fluids is rarely contributive; cultures are long, and not cost effective [5] [7] [8] [10]. The GeneXpert is a sensitive tool that allows a rapid detection of bacilli within hours, but a negative result does not exclude TB [25]. In our study the KB was isolated in 7\% of cases.

The histological study was done in five of our patients; it had confirmed the diagnosis in two lymph node biopsies and autopsies. Some authors suggest the use of invasive procedures with tissue biopsy, because it helps to confirm the diagnosis, to start early treatment, and to have a good outcome [11] [15] [18]. In addition to the poor specificity of symptoms, also the site of TB is often extra-pulmonary. In the literature, this frequency varies from $47 \%$ to $100 \%$; the lymph node and peritoneal tuberculosis are the most common sites [3] [5] [9] [11] [15]. In our study, the extrapulmonary location was predominant (79\%). All the authors reported the difficulty in the diagnosis of TB in the CHD; it is due to non-specific symptoms and the frequency of extrapulmonary location [3] [5] [7] [9] [11] [15]. In our series sure diagnosis was only achieved in $17 \%$ of patients, $7 \%$ bacteriology, $7 \%$ on tissue biopsy histology and 3\% at autopsy.

Some authors recommend starting a probabilistic TB treatment without formal proof of TB in CHD based on strong presumptive arguments. The favorable response to treatment will later confirm the diagnosis [3] [5] [8] [9]. The treatment of TB in the CHD is not codified. The protocols and duration of antituberculosis treatment vary according to the authors. Some associate RHZ [11] [15], others RHZE [5] [10] [11] for the first two months, and HR during the maintenance phase. Dosages of $\mathrm{R}$ and $\mathrm{H}$ should not be changed, while the $\mathrm{Z}$ and $\mathrm{E}$ are respectively administered at the dose of $30 \mathrm{mg} / \mathrm{kg}$ and $20 \mathrm{mg} / \mathrm{kg}$ every 48 hours. Streptomycin is given at a dose of $750 \mathrm{mg}$ every 72 hours, six hours before each dialysis with close monitoring of serum. [3] In the Senegalese TB program, this drug is reserved for retreatment protocols.

Because of their inability to excrete chemicals, side effects of TB drugs are commonly observed in the CHD [4]. Isoniazid was the most offending drug, it was responsible for $53 \%$ of neuropsychiatric effects in our series, against 100\% in Niang studies [3], 54\% in Yao studies [10] 43\% in studies of Sen [9] and Quantrill. [4] These effects can be avoided by administering pyridoxine to $100 \mathrm{mg}$ daily [3]. The retrobulbar optic neuritis was re- 
ported in Sen [9] and Yao [10] studies with respectively $43 \%$ and $46 \%$ of cases. Visual acuity and color vision before and regularly during treatment and control serum level are also recommended [26]. The incidence of drug-induced hepatitis is very low in our serie. Compared with Turkish serie, N Sen found 3 cases of hepatotoxicity among 18 patients [9]. Only early detection and adequate treatment can guarantee a good prognosis. The combination of immune deficiency, malnutrition and tuberculosis infection increases the risk of tuberculosis and mortality [3] [9] [11]. The death rate ranges from $0 \%-75 \%$ according to studies [8]-[11] [15]. It is often attributed to delayed diagnosis and treatment. In our study, the outcome was favorable in $72 \%$ of cases. We noted the death of 6 patients (21\%). Mortality was attributed to disseminated tuberculosis in 50\% of cases. This result is superimposed on that found in Mali (70\% of favorable evolution) [8], and better than that reported in Ivory Coast 57\% [10]. However a better evolution was reported in Turkey [9] and Brazil [15] with respective recovery rates of $94.5 \%$ and $97 \%$.

Some authors recommend early treatment of latent tuberculosis infection in chronic hemodialysis patients to prevent progression to disease state [6] [9]. Anti LAM (Antilipoarabinomannan Antibody) antibody is sometimes useful to diagnose latent tuberculosis which can help to start treatment earlier [27]. The drug of choice is $\mathrm{H}$ which can be administered 2 to 3 times a week under the supervision of the dialysis team, at a dose of $15 \mathrm{mg} /$ $\mathrm{kg} /$ dose for a period of 6 to 9 months, associated with pyridoxine $100 \mathrm{mg} / \mathrm{j}$ [4]. Chemotherapy is not devoid of $\mathrm{SE}$; it requires close monitoring [4]. In our series no cases of latent tuberculosis infection was sought.

\section{Conclusion}

TB among chronic hemodialysis is more common compared to the general population because of immune dysfunction associated with chronic kidney disease. Early diagnosis and treatment are the guarantee of a good outcome.

\section{References}

[1] Man, N.K., Touam, M. and Jungers, P. (2010) Hemodialysis for Renal Replacement Therapy. Lavoisier SAS, 56-61, 112-116.

[2] Kato, S., Chmielewski, M., et al. (2008) Aspects of Immune Dysfunction in End-Stage Renal Disease. Clinical Journal of the American Society of Nephrology, 3, 1526-1533. http://dx.doi.org/10.2215/CJN.00950208

[3] Niang, A., Diouf, B., Leye, A., Ndiaye, F.S. and Diop, T.M. (2005) Diagnostic and Therapeutic Characteristics of Tuberculosis in Chronic Hemodialysis Patients in Dakar. Medecine Tropicale, 65, 49-52.

[4] Quantrill, S.J., Woodhead, M.A., Bell, C.E., Hardy, C.C., et al. (2002) Side-Effects of Antituberculosis Drug Treatment in Patients with Chronic Renal Failure. European Respiratory Journal, 20, 440-443. http://dx.doi.org/10.1183/09031936.02.00298002

[5] Hassine, E., Marniche, K., Hamida, J., Hassine, K., Bouaziz, A. and Ben Mustapha, M.A. (2002) Tuberculosis of Hemodialysis Patients in Tunisia. Nephrology, 23, 135-140.

[6] Chagas, A.C.F., Filho, G.H., et al. (2014) Prevalence of Latent Tuberculosis and Treatment Adherence among Patients with Chronic Kidney Disease in Campo Grande, State of Mato Grosso do Sul. Revista da Sociedade Brasileira de Medicina Tropical, 47, 204-211. http://dx.doi.org/10.1590/0037-8682-0035-2014

[7] Manmadha Rao, T., Ram, R., Swarnalatha, G., Santhosh Pai, B.H., Ramesh, V., et al. (2013) Tuberculosis in Haemodialysis Patients: A Single Center Experience. Indian Journal of Nephrology, 23, 340-345. http://dx.doi.org/10.4103/0971-4065.116296

[8] Fongoro, S., Diallo, D., Toloba, Y., Diallo, S., et al. (2011) The Case of Tuberculosis to the Chronic Hemodialysis Patients at the Teaching Hospital of Point G (CHU) in Bamako about 10 Remarks. Mali Medical, XXVI, 27-30.

[9] Sen, N., Turunc, T., Karatasli, M., Sezer, S., Demiroglu, Y.Z. and Eyuboglu, O. (2008) Tuberculosis in Patients with End-Stage Renal Disease Undergoing Dialysis in an Endemic Region of Turkey. Transplantation Proceedings, 40, 8184.

[10] Yao, K.H., Bakayoko, A., Henyo, F., et al. (2011) Particularities of Tuberculosis in Black Africans Chronically Dialysed. Néphrologie \& Thérapeutique, 7, 242-244.

[11] El Kabbaj, D., Bahadi, A. and Oualim, Z. (2010) Prevalence of Tuberculosis in Hemodialysis Patients. Saudi Journal of Kidney Diseases and Transplantation, 21, 164-167.

[12] Klote, M.M., Agodoa, L.Y. and Abbott, K.C. (2006) Risk Factors for Mycobacterium tuberculosis in US Chronic Dialysis Patients. Nephrology Dialysis Transplantation, 21, 3287-3292. http://dx.doi.org/10.1093/ndt/gfl488 
[13] Inoue, T., Nakamura, T., Katsuma, A., et al. (2009) The Value of QuantiFERON® TB-Gold in the Diagnosis of Tuberculosis among Dialysis Patients. Nephrology Dialysis Transplantation, 24, 2252-2257. http://dx.doi.org/10.1093/ndt/gfp030

[14] Wauters, A., Peetermans, W.E., Van den Brande, P., et al. (2004) The Value of Tuberculin Skin Testing in Hemodialysis Patients. Nephrology Dialysis Transplantation, 19, 433-438. http://dx.doi.org/10.1093/ndt/gfg569

[15] Marques, L.P.J., Rioja, L.S., Pacheco, G.G.L.C., et al. (2008) Tuberculosis in Hemodialysis Patient in Area of High Incidence of Mycobacterium tuberculosis and Human Immunodeficiency Virus Infection. Dialysis \& Transplantation, 37, 486-490. http://dx.doi.org/10.1002/dat.20275

[16] Hussein, M.M., Mooij, J.M. and Roujouleh, H. (2003) Tuberculosis and Chronic Renal Disease. Seminars in Dialysis, 16, 38-44. http://dx.doi.org/10.1046/j.1525-139X.2003.03010.x

[17] Lee, C.-T., Hung, K.-H., Lee, C.-H., et al. (2002) Chronic Hypercalcemia as the Presenting Feature of Tuberculous Peritonitis in a Hemodialysis Patient. American Journal of Nephrology, 22, 555-559. http://dx.doi.org/10.1159/000065264

[18] Hung, Y.-M., Chan, H.-H. and Chung, H.-M. (2004) Tuberculous Peritonitis in Different Dialysis Patients in Southern Taiwan. American Journal of Tropical Medicine and Hygiene, 70, 532-535.

[19] Segall, L. and Covic, A. (2010) Diagnosis of Tuberculosis in Dialysis Patients: Current Strategy. Clinical Journal of the American Society of Nephrology, 5, 1114-1122. http://dx.doi.org/10.2215/CJN.09231209

[20] Agarwal, S.K., Gupta, S., Bhowmik, D. and Mahajan, S. (2011) Reaction Size in Tuberculin Test Positivity in Hemodialysis Patients-Author's Reply. Indian Journal of Nephrology, 21, 139-140. http://dx.doi.org/10.4103/0971-4065.82381

[21] Savaj, S., Savoj, J., Ranjbar, M. and Sabzghabaei, F. (2014) Interferon-Gamma Release Assay Agreement with Tuberculin Skin Test in Pretransplant Screening for Latent Tuberculosis in a High-Prevalence Country. Iranian Journal of Kidney Diseases, 8, 329-332.

[22] Maden, E., Bekci, T.T., Kesli, R., Atalay, H., et al. (2011) Evaluation of Performance of Quantiferon Assay and Tuberculin Skin Test in End Stage Renal Disease Patients Receiving Hemodialysis. New Microbiologica, 34, 351-356.

[23] Al Jahdali, H., Ahmed, A.E., Balkhy, H.H., et al. (2012) Comparison of the Tuberculin Test Skin and Quanti-FeronTB Gold In-Tube (QFT-G) Test for the Diagnosis of Latent Tuberculosis Infection in Dialysis Patients. Journal of Infection and Public Health, 6, 166-172. http://dx.doi.org/10.1016/j.jiph.2013.02.002

[24] Gobert, D., Lidove, O., de Fontbrune, F.S., Peltier, J., et al. (2007) Adenosine Deaminase Is Useful for the Diagnosis of Peritoneal Tuberculosis in Patients with End-Stage Renal Failure. Review of Medicine, 28, 507-509.

[25] Richardson, R.M.A. (2012) The Diagnosis of Tuberculosis in Dialysis Patients. Seminars in Dialysis, 4, 419-422. http://dx.doi.org/10.1111/j.1525-139X.2012.01093.x

[26] Fang, J.-T., Chen, Y.-C. and Chang, M.-Y. (2004) Ethambutol-Induced Optic Neuritis in Patients with End Stage Renal Disease on Hemodialysis: Two Case Reports and Literature Review. Renal Failure, 26, 189-193. http://dx.doi.org/10.1081/JDI-120038521

[27] Eleftheriadis, T., Tsiaga, P., Antiniadi, G., et al. (2005) The Value of Serum Antilipoarabinomannan Antibody Detection in the Diagnosis of Latent Tuberculosis in Hemodialysis Patients. American Journal of Kidney Diseases, 46, 706712. http://dx.doi.org/10.1053/j.ajkd.2005.06.021 